\title{
Mitrei del Vicino Oriente: UnA faCiES ORIENTALE DEL CULTO MISTERICO DI MITHRA
}

\author{
Tommaso Gnoli \\ Università di Bologna
}

\begin{abstract}
An Oriental facies of the cult of Mithras is testified in the Roman East from the first century onward. This article proposes evidence and discusses crucial and peculiar characteristics and idiosyncrasies in the known mithraea from the Roman Near East, against the generally accepted theory stating that the cult of Mithras in the East, and particularly in Syria, shared the same features as those known of Mithraism in the West.
\end{abstract}

Key words: Mithras, Syria, tauroctony, oriental cults, Iranian religion.

Un argomento largamente utilizzato per opporsi alla teoria diffusionista relativa al culto di Mithra in Occidente, propugnata con forza persuasiva da Franz Cumont a partire dai grandi capolavori che egli dedicò all'argomento tra otto e novecento, ${ }^{1}$ era costituito dalla scarsità delle attestazioni del culto misterico di Mithra nell'Oriente romano, in Siria, Giudea, Arabia, oltre che nella penisola Anatolica. Quando Cumont scriveva le sue opere, da queste regioni le testimonianze del culto misterico di Mithra erano molto scarse e per lo più piuttosto tarde. La situazione era quindi caratterizzata da un certo numero di fonti letterarie, che affermavano un'origine orientale del culto, che avrebbe assunto la sua forma misterica caratteristica nell'impero Romano in Cilicia già in epoca tardorepubblicana - si pensi soprattutto a Plutarco - e le testimonianze archeologiche che, al contrario, mostravano una certa abbondanza di monumenti soprattutto nell'Occidente europeo e in Italia, comunque mai prima degli ultimi decenni del primo secolo dopo Cristo. Nemmeno l'importante scoperta del mitreo di Dura Europos, avvenuta all'inizio di Febbraio del 1934, poté essere utilizzata per sostanziare le tesi di Cumont di una derivazione orientale del culto misterico di Mithra. ${ }^{2}$ La data relativamente tarda del mitreo, non anteriore alla conquista del sito da parte di Roma sotto gli auspici di Lucio Vero, non consentiva di utilizzare in questo senso la pur rilevante scoperta, e Cumont non mancò

\footnotetext{
1 Cumont 1896-1899; 1902²; 1906.

2 Cumont 1975, 151-214; cfr. ora Gnoli 2016, 146-164.
} 
di manifestare la sua contrarietà dopo l'iniziale entusiasmo, dovuto anche al fatto che lo studioso belga aveva precedentemente scritto al suo collega Mikhail Ivanovič Rostovtzeff che si sarebbe aspettato proprio di rinvenire un tempio dedicato a Mithra nella città recentemente scoperta. ${ }^{3}$

Nonostante le sopra evidenziate difficoltà di una documentazione che non la supportava pienamente, la teoria cumontiana non faticò comunque ad imporsi presso un pubblico che andava molto al di là degli specialisti studiosi di Storia antica, e il culto misterico di Mithra finì con il rappresentare l'esempio più noto di quel sincretismo religioso che avrebbe caratterizzato il paganesimo romano, prima che la fede di Roma si polarizzasse sugli assoluti ed esclusivi dogmi cristiani. Dopo tutto i seguaci del dio vestito alla persiana non si definivano essi stessi seguaci di una religione persiana, e non chiamavano Persae alcuni loro compagni, giunti a un certo livello d'iniziazione?

Contemporaneamente alla morte di Cumont, subito dopo la fine del secondo conflitto mondiale, le tesi dello studioso belga vennero frontalmente aggredite da importanti lavori di un iranista svedese, Stig Wikander (1908-1983), che smantellò dalle fondamenta le convinzioni che avevano indotto Cumont a definire il culto misterico di Mithra come la forma romana del mazdeismo iranico. L'importanza dei lavori di Wikander e la loro genesi anche in rapporto alla netta rivalità personale, oltre che scientifica, che l'oppose al suo collega Geo Widengren, meriterebbero un'analisi approfondita, fatto sta che fino a tempi recentissimi i suoi lavori, e in particolare Der arische Männerbund (1938), Feuerpriester in Kleinasien und Iran (1946), Études sur les mystères de Mithras (1950), divennero gli unici lavori di iranisti noti e citati dagli studiosi del mondo classico che intendevano affrontare il problema dei culti misterici di Mithra in modo autonomo rispetto al 'sistema cumontiano', come si può facilmente evincere, ad esempio, dalla influente sintesi di Martin Persson Nilsson, ${ }^{4}$ perfettamente contemporanea all'ultimo lavoro citato di Wikander.

Nel frattempo le cose si erano mosse, e molto, anche tra gli studiosi del mondo romano. Il lavoro più importante e influente in questo campo fu un articoletto di appena sei pagine, pubblicato nel 1937 sul Journal of Roman Studies da Arthur Darby Nock, dal titolo The Genius of Mithraism.

La grande ripresa degli studi mitraici che ha avuto luogo negli anni 1970, grazie anche alla sponsorizzazione dello scià Reza Pahlavi, ${ }^{5}$ vide oramai costituiti due precisi schieramenti, che si potrebbero schematicamente definire come il vecchio paradigma ermeneutico, che faceva capo all'autorità incontrastata di Cumont e all'epoca incarnato soprattutto dal suo allievo Maarten Vermaseren, ${ }^{6} \mathrm{e}$ un nuovo paradigma che, prendendo le mosse dai lavori di Wikander, si sostanziava nel campo degli studi classici nelle posizioni di Richard Gordon e in quelle ancora più estreme di Roger Beck. ${ }^{7}$ Naturalmente neanche il più 'iranizzante' tra gli studiosi filo-cumontiani sarà stato più disposto ormai a sottoscrivere l'aforisma del mitraismo occidentale come una forma romana di mazdeismo, ma ciò che rimaneva, a vari livelli, della ricostruzione di Cumont, era la derivazione iranica delle idee religiose che stavano dietro una pratica cultuale che, con ogni

\footnotetext{
${ }^{3}$ Bongard-Levin et al. 2007, 192-193, n 104-105; Gnoli 2016, 145-146.

${ }^{4}$ Nilsson 1950, 666 sgg.

${ }^{5}$ Hinnels 1975; Duchesne-Guillemin 1978; Bianchi 1978; Journal of Mithraic Studies.

${ }^{6}$ Vermaseren 1956 (=CIMRM); 1963; 1971; 1974; 1982.

${ }^{7}$ Gordon 1975, 215-248; Beck 1984, 2002-2115; 2000, 145-180; 2004a; 2006.
} 
evidenza, si era profondamente arricchita e nutrita in ambienti culturali ellenistici, che avevano nell'Asia Minore, e più specificamente nell'area compresa tra la Commagene e Tarso - ricordiamoci la Cilicia di Plutarco (Pomp. 24,7) - il loro focus principale, negli ultimi decenni dell'evo antico. Dall'altra parte, invece, stavano i fautori di ipotesi in varia misura opposte: nella forma più estrema non esisterebbe alcun legame tra il Mithra iranico o vedico e il mitraismo romano; quest'ultimo, piuttosto, è stato un'invenzione del tutto romana, che sarebbe stata creata da un brillante astrologo attivo nella Roma dell'età neroniana. Costui si sarebbe servito, per la sua creazione, di un confuso immaginario iranizzante, che sarebbe stato corrente negli ambienti di corte raffinati e orientaleggianti del filelleno imperatore romano. Si è creduto pertanto di poter persino dare un nome a questo Genius of Mithraism: Ti. Claudius Balbillus. ${ }^{8}$

A partire dagli anni '70 il dibattito si è andato via via chiarendo e nello stesso tempo definendo, acquisendo però caratteristiche sempre più disciplinari: tra gli orientalisti le posizioni di Wikander apparvero fin da subito estreme e non prive di esagerazioni e inesattezze, mentre tra gli studiosi del mondo romano queste continuano ancora oggi ad essere utilizzate in via preferenziale perché funzionali al nuovo paradigma.

Tutto quanto è stato scritto finora non è finalizzato a scrivere una spigolatura sulla storia delle ricerche sul mitraismo nel mondo antico, ma piuttosto per tratteggiare il quadro all'interno del quale si colloca il dibattito sullo specifico tema del mitraismo in Siria e più in generale nel Vicino Oriente romano, e cercare di chiarire perché questo tema sia stato ritenuto così importante da aver indotto Marie-Françoise Boussac e PierreLouis Gatier ad organizzare nel 2000 un convegno internazionale specificamente dedicato al tema. Gli atti di quel Convegno vennero poi pubblicati sul volume 11 della rivista Topoi, targato 2001 ma apparso in realtà nel 2003.

Da allora le nostre conoscenze di monumenti mitraici provenienti dal Vicino Oriente non si sono accresciute. La base documentaria è rimasta la stessa. Ciò che però colpisce nella presentazione di alcuni di quei nuovi monumenti ritrovati nel corso del ventesimo secolo un po' in tutto il Vicino Oriente - certamente in numero enormemente ridotto rispetto ad altre regioni di confine dell'impero - è il tentativo di far necessariamente rientrare tali scoperte nel nuovo paradigma ermeneutico sottovalutando tutto quanto in esse poteva puntare verso direzioni differenti. Gioverà citare ciò che, a questo riguardo, Lewis Hopfe scrisse a conclusione della sua analisi del Mithraism in Syria in un articolo comparso nel 1990 in Aufstieg und Niedergang der römischen Welt:

the form of Mithraism which was practiced between the second and the late third centuries CE in Syria was not native to that area. Again, from rather meagre evidence, it would appear that the Mithraism of Syria was in style and form identical to that which was practiced in the western portion of the Empire. The notion that Mithraism was founded in Persia and moved westward toward Rome, simply cannot be sustained by our study. The iconography of Mithraism in Syria is so similar to that which appears in the west that it can only have been transported to Syria by Romans. ${ }^{9}$

${ }^{8}$ È la tesi estrema di R. Merkelbach 1984, solo parzialmente ammorbidita da R. Beck 1998, 115-128, in partic. 126-127 = Beck 2004a, 31-44, in partic. 43; Beck 2004b = Beck 2004a, 323-329. A mio parere, nonostante la bibliografia sopra citata, mi sembra che rimanga insostituibile, per l'argomento, il lavoro di J. Gagé 1968. La questione relativa alla personalità di Balbillus è estremamente intricata, ma è certo che le speculazioni sul suo ruolo nella 'creazione' del mitraismo poggiano su identificazioni quanto meno incerte. Su questi professionisti a metà tra sacro e magia è ora uscita la monografia di $\mathrm{H}$. Wendt 2016 (non vidi).

${ }^{9}$ Hopfe 1990, 2216-2234, in partic. 2233. 
In questo contributo si vuol presentare quanto vi sia di originale in una selezione (arbitraria) di monumenti mitraici dal Vicino Oriente, sia che tale originalità sia comodamente inquadrabile all'interno del nuovo paradigma, sia che invece presenti elementi che appaiono molto più a loro agio nell'ambito del vecchio paradigma ermeneutico, senza con questo voler prendere posizione sui tanti problemi che ancora circondano questo culto misterico, ma semplicemente con l'intento di ampliare la fenomenologia presente nella documentazione, valorizzando elementi finora sottovalutati.

\section{La cronologia}

Come si è potuto vedere, al problema della cronologia dei monumenti mitraici del Vicino Oriente è stata data spesso molta importanza, nonostante sia evidente che non possa comunque mai esser considerato decisivo: l'attestazione datata di un tempio mitraico non può mai essere indizio sicuro della data d'introduzione del culto, ma solamente un terminus post quem. Tuttavia uno dei punti sempre messi in rilievo nell'ambito del nuovo paradigma è costituito dalla pretesa recenziorità delle attestazioni orientali rispetto a quelle occidentali, e romane in particolare. Ora, questo assunto non è vero. Nonostante la ristrettezza dei dati in nostro possesso - i mitrei noti in tutto il Vicino Oriente sono appena sette - tre, il mitreo rupestre di Doliche e quello di Caesarea Maritima, in Israele e quello ipogeo di Hūarte, sono molto antichi, e anche un mitreo scoperto in Trachonitis sembra poter datare ai primissimi decenni del secondo secolo, benché i dati che da quest'ultimo possono trarsi siano da questo punto di vista veramente esigui.

Il complesso cultuale rinvenuto nei pressi di Doliche è senza mezzi termini imbarazzante per il nuovo paradigma. Si tratta di un mitreo doppio ricavato in una grotta naturale, che è stata appena adattata per poter servire allo scopo. ${ }^{10}$ L'ingresso è costituito da un cunicolo piuttosto angusto che immetteva in due camere sotterranee, che sembra siano state approntate in periodi diversi ma che vennero utilizzate entrambe per un periodo di tempo abbastanza lungo. Il luogo era stato scoperto già in antico ed è stato fatto oggetto dello zelo iconoclasta di visitatori cristiani, che hanno sfregiato una delle due tauroctonie con il segno della croce. Tutti gli arredi e le sculture sono stati accuratamente distrutti, dai cristiani e dai cercatori di tesori, e quel che oggi è in qualche modo ricostruibile sono solamente le due tauroctonie che, una per ogni sala cultuale, sono oramai leggibili solamente in negativo, segnate dai colpi degli scalpelli. Da quanto è possibile ricostruire si tratta di immagini consuete, dove sono presenti tutti i protagonisti abituali di queste affollate iconografie. Lo stato dei rilievi non consente di dire nulla sullo stile delle sculture e quindi di trarre qualche pur esile indicazione cronologica dai rilievi. Un'iscrizione, che avrebbe certamente fornito elementi molto utili, posta nell'ingresso tra le due camere cultuali, è stata così accuratamente abrasa da risultare oggi del tutto illeggibile.

Nonostante l'accurata distruzione del complesso ipogeo, vi sono state ritrovate tre monete di bronzo. Se non aggiungono molto alle nostre ansie cronologiche i due bronzi di Elagabalo, ben diverso è il discorso per quanto riguarda un bronzo di Antioco IX,

10 Schütte-Maischatz - Winter 2001, 149-173; Schütte-Maischatz - Winter 2004; con la recensione molto critica di R.L. Gordon (2007, 602-610). 
emesso dalla zecca di Antiochia di Siria tra il 114 e il 112 a.C. La moneta è stata trovata in buone condizioni, priva dei segni di usura di una prolungatissima circolazione. È evidente che non può esser giunta in quella grotta se non al massimo alla fine del I secolo a.C. Nulla in quanto resta nelle grotte autorizza a supporre un uso dei locali precedente a quello testimoniato dalle distrutte tauroctonie. L'esigenza di ipotizzare un impiego precedente del complesso deriva solamente dalla scarsa integrazione di questa scoperta all'interno del nuovo paradigma ermeneutico.

Anche il mitreo di Caesarea Maritima è molto antico. ${ }^{11}$ Anche qui la situazione è molto complessa e non sembra facile riuscire a determinare con esattezza quando una piccola parte dei magazzini della rocca erodiana nei pressi del porto della grande colonia romana venne adibita a mitreo. Sembra tuttavia che aree immediatamente adiacenti al mitreo siano state rifunzionalizzate in età neroniana, e solo la scarsa adesione di questo dato al nuovo paradigma ermeneutico induce ad immaginare una datazione per il mitreo in una data compresa fra Vespasiano e Traiano, secondo la formulazione di Gordon. ${ }^{12}$ Anche con questa datazione per così dire prudente, il mitreo di Caesarea Maritima si colloca tra le primissime attestazioni del culto misterico di Mithra in tutto il mondo romano.

I dati cronologici desumibili dal mitreo di Ḥūarte sembrano meno decisivi. ${ }^{13}$ Oggi si può ragionare solamente sulla fase di metà quarto secolo, ma Michał Gawlikowski ha potuto rilevare una lunghissima utilizzazione del complesso, la cui utilizzazione potrebbe risalire addirittura alla seconda metà del I secolo d.C. ${ }^{14}$

Insomma, dei sette mitrei noti nel Vicino Oriente romano, tre, quello doppio di Doliche, quello di Caesarea, e quello di Ḥūarte, si candidano ad essere tra i più antichi mitrei finora noti. Uno, Doliche, addirittura sarebbe molto più antico di quanto ammesso dal nuovo paradigma per la data d'invenzione del culto misterico di Mithra da parte di Balbillo, anzi in una data molto vicina a quella plutarchea per l'introduzione del culto misterico in Cilicia, sulla quale molto si basava il vecchio paradigma.

\section{La sintassi della tauroctonia}

L'immagine cultuale centrale nel culto misterico di Mithra è la tauroctonia. Mithra è collocato all'interno di una grotta - quasi sempre rappresentata come un antro naturale mentre accoltella sulla spalla un grande toro bianco. La posa in cui è ritratto il dio è sem-

11 Bull 1978, 75-89; Blakely 1987.

12 Gordon 2001a, 77-136, in partic. 81: "Une pièce néronienne trouvée dans la phase céramique 4 indiquerait, jointe à d'autres indications de datation absolue, que le magasin fut réaménagé en temple au plus tard sous Domitien. Le mithreum appartiendrait alors au petit groupe de temples et inscriptions, échelonnés de l'aire danubienne jusqu'à Rome ... qu'on date de l'époque flavienne-trajanienne (80-120 d.n.è.)." Non sfuggerà la forzatura presente in questa affermazione: i dati archeologici sembrerebbero rinviare a una datazione "au plus tard sous Domitien", cioè ben addentro al primo secolo, non al secondo.

13 Gawlikowski 2000, 161-171; Parandowska 2002, 295-299; Majcherek 2003, 325-334; Chabiera Parandowska - Troclimowicz 2004, 321-326; Gawlikowski 2007, 337-361.

14 Gli strati di intonaco dipinto certamente individuabili sono almeno cinque nella cella: Gawlikowski 2007, in partic. 342. La lunghissima utilizzazione del mitreo è però testimoniata con certezza dal vasellame a da altri ritrovamenti: Gawlikowski 2014, 109-117, in partic. 111: "the Mithraic use of the cave at Hawarte would be at least contemporary to the earliest Italian monuments of this cult." 
pre la stessa, con piccole varianti che non sembrano significative: Mithra è disposto sul groppone dell'animale ormai inginocchiato ventre a terra, mentre con la sinistra solleva la testa del toro afferrandolo per le narici (molto più raramente per le corna), con la destra affonda il coltello nel collo dell'animale. La gamba destra del dio è distesa all'indietro e il più delle volte immobilizza la zampa posteriore destra dell'animale fermandola a terra, mentre la gamba sinistra è piegata all'altezza della spalla dell'animale, a poca distanza dal punto della ferita. La testa del dio è (quasi) sempre girata all'indietro, guardando nella direzione esattamente contraria rispetto al sacrificio, dalla coda dell'animale morente spesso germogliano spighe di grano. Accanto a questa rappresentazione, che è il fulcro centrale dei misteri, vi sono una pletora di personaggi secondari, che agiscono e assistono in vario modo all'azione cruenta, e compaiono secondo una sintassi che conosce poche variazioni significative.

La scena che, sulla base del numero delle testimonianze conservate, si potrebbe considerare l'archetipo sul quale si sono poi create tutte le numerose eccezioni, prevede che il dio Mithra sia vestito alla persiana, con pantaloni, cintura, foderi di pugnali (per lo più due), mantello e berretto frigio. Laddove la scena è conservata in pittura i colori più comunemente attestati sono un abito di Mithra sul rosso, il mantello sempre blu. Sul mantello molto spesso sono rappresentati sette corpi celesti. Ad interagire direttamente con l'uccisione del toro, all'interno della grotta, sono raffigurati un corvo, che svolazza in alto, vicino alla testa del dio, mentre in basso, sul pavimento della grotta, accanto al corpo della bestia agonizzante, sono rappresentati uno scorpione attaccato ai testicoli del toro, un cane e un serpente che leccano la ferita sanguinante. Talvolta il serpente è rappresentato attorcigliato a un cratere. Talvolta il cratere è abbandonato a se stesso, non di rado è assente. Qualche volta si vede anche un leone, sempre in posizione molto secondaria, sullo sfondo.

Al di fuori della grotta, normalmente a destra e a sinistra della scena e spesso con la funzione compositiva di delimitare con la loro presenza lo spazio sacro della tauroctonia, vi sono i due dadofori rappresentati in un ordine che è molto difficile stabilire con certezza, se originariamente a destra o a sinistra: Cautes e Cautopates. Sempre al di fuori della grotta vi sono infine, agli angoli superiori dello Urtypus,${ }^{15}$ Sol e Luna, rappresentati per lo più come busti all'interno di due medaglioni e per i quali pure si ha la stessa capricciosa alternanza in quanto a posizione così come avviene per i due dadofori. Le aggiunte più comuni rispetto a questa raffigurazione, che segnalo perché a mio parere potrebbero essere piuttosto antiche, ma che non mi sembra possibile abbiano fatto parte dell'archetipo, sono:

1) la presenza dello zodiaco sull'arco che delimita la volta della grotta;

2) la presenza di una figura barbata con kalathos in posizione centrale, nel bel mezzo dello zodiaco;

3) la presenza di sette stelle oppure di sette cilindri (quasi unanimemente intesi come altari) allineati in varia posizione, ma sempre all'esterno della grotta. Quando si tratta di sette stelle, la posizione di questa successione è sempre in alto, al centro, fuori

15 Dato l'alto numero di varianti esistenti tra le varie tauroctonie è molto difficile determinare con precisione lo Urtypus. Ritengo siano ancora molto utile le analisi tipologiche condotte sulla scena da L.A. Campbell (1968). Le modifiche che da allora si siano rese necessarie sulla base soprattutto di nuovi ritrovamenti non mi sembra che tolgano validità al metodo di catalogazione delle varianti della tauroctonia. 
dalla grotta e in posizione simmetrica rispetto alla composizione. Mi sembra piuttosto evidente che questi elementi, benché piuttosto comuni, non possano essere intesi come elementi originali, bensì delle esplicitazioni del senso recondito di questa rappresentazione.

È stato mostrato, a mio parere in modo convincente, ${ }^{16}$ che tale senso recondito fosse una mappa astrale, e che proprio a partire da questa mappa si sia costituita questa raffigurazione complicatissima e affascinante, che quindi viene ripetuta senza eccessive differenze in tutti i luoghi dell'impero romano dove è attestato il culto misterico di Mithra.

Le tauroctonie siriane non contravvengono a questa sintassi generale, imposta da un elemento con ogni evidenza centrale nel culto del dio persiano e ovunque ripetuta, ma si segnalano per una particolarità compositiva che è opportuno rilevare. In una percentuale consistente rispetto al totale delle tauroctonie conosciute nel Vicino Oriente si ha l'inserzione, all'interno dello spazio mistico dove ha luogo il sacrificio, di elementi normalmente relegati all'esterno della rappresentazione principale. Non è raro, infatti, che accanto alla scena centrale dell'uccisione del toro siano rappresentate anche altre scene relative a un mito che doveva essere certamente molto complesso e che risulta oggi difficilissimo da ricostruire. ${ }^{17}$ Queste scene, difficilmente del tutto ripetitive ma, appunto, ricche di variazioni anche importanti, sono però quasi sempre relegate al di fuori di quell'area delimitata dalla volta della grotta, spesso relegate in pannelli disposti sopra, sotto o ai lati della scena centrale, che risulta comunque nettamente delimitata nello spazio e distinta.

Ora, nella maggior parte delle tauroctonie dal Vicino Oriente questo spazio nel quale avviene la tauroctonia e che ammette la presenza solamente delle figure che abbiamo già elencato, risulta ingombro di figure e di scene che, quando hanno riscontri, sono normalmente all'esterno della grotta. Pur lasciando da parte la tauroctonia da Arsha-waQibar, fuori da qualsiasi standard per molti motivi (CIMRM 71), ${ }^{18}$ anche la tauroctonia proveniente da Sidone e oggi a Parigi (CIMRM 75, fig. 1) è caratterizzata da una sintassi che non può non colpire lo spettatore esperto di arte mitraica: tutto attorno alla scena del sacrificio, molto ben equilibrata, stanno, senza alcun elemento di campitura rappresentato da grotte o altro, tutti i segni dello zodiaco, ai quali si frammischiano ben sei tondi con busti, quattro in alto e due in basso. I medaglioni posti ai quattro angoli rappresentano le stagioni (da in basso a sinistra inverno, primavera, estate, autunno), mentre i due

${ }^{16}$ Gnoli 2010, 77-86.

${ }^{17}$ Non è possibile fornire qui indicazioni anche solo generali sui numerosissimi tentativi di ricostruzione della mitologia mitraica. La loro elencazione coinciderebbe sostanzialmente con la bibliografia relativa al mitraismo stesso. Segnalo comunque l'ultimo tentativo del quale sono a conoscenza, che si raccomanda quanto meno per accuratezza e completezza: Mastrocinque 2017. Eppure, recentemente, una nuova linea interpretativa del culto di Mithra si sta affermando che tenderebbe a negare l'esistenza stessa di un mito mitraico: Martin 2005, 187-217; Beck 2014, 72-89. I problemi sollevati da questi e altri studi sulla medesima falsariga esulano dallo scopo di queste pagine. Spero di potermene occupare altrove.

${ }^{18}$ Non sono mai riuscito a trovare un'immagine dettagliata di questo pezzo pur così importante. L'immagine pubblicata da Vermaseren in CIMRM riproduce la cattiva fotografia pubblicata già da F. Cumont (1933, 381-395) e non consente di individuare eventuali dettagli presenti in questo monumento, che sembra comunque estremamente rozzo, inciso su un calcare locale di scarsa qualità da scultori inesperti. Il pezzo venne ritrovato da un militare francese nel 1932 e pubblicato fuori contesto nel citato studio di Cumont. Recentemente P.-L. Gatier (2001, 175-182), descrive il sito e lo contestualizza, ma con speciale riferimento ad un altro reperto, che non ha connessioni con il culto di Mithra. 
medaglioni più centrali in alto rappresentano Sol (a destra) e Luna (a sinistra). Non c'è nulla in questa raffigurazione di straordinario se non la mancanza di qualsiasi contesto naturalistico, che crea un obiettivo disordine generale della composizione, che risulta così proiettata in un mondo immateriale e astratto, popolato da segni astrali, di indubbia suggestione. L'unico altro esempio che conosco che fa ricorso a un così alto grado di astrazione è una tauroctonia proveniente dal mitreo di Walbrook, Londra, e oggi conservata al Museum of London (CIMRM 810). Da notare, infine, l'assenza dei dadofori, che invece nella rozza immagine da Arsha-wa-Qibar avevano un ruolo importantissimo nel campire l'immagine stessa e risultavano di dimensioni addirittura molto maggiori rispetto a quelle dello stesso Mithra.

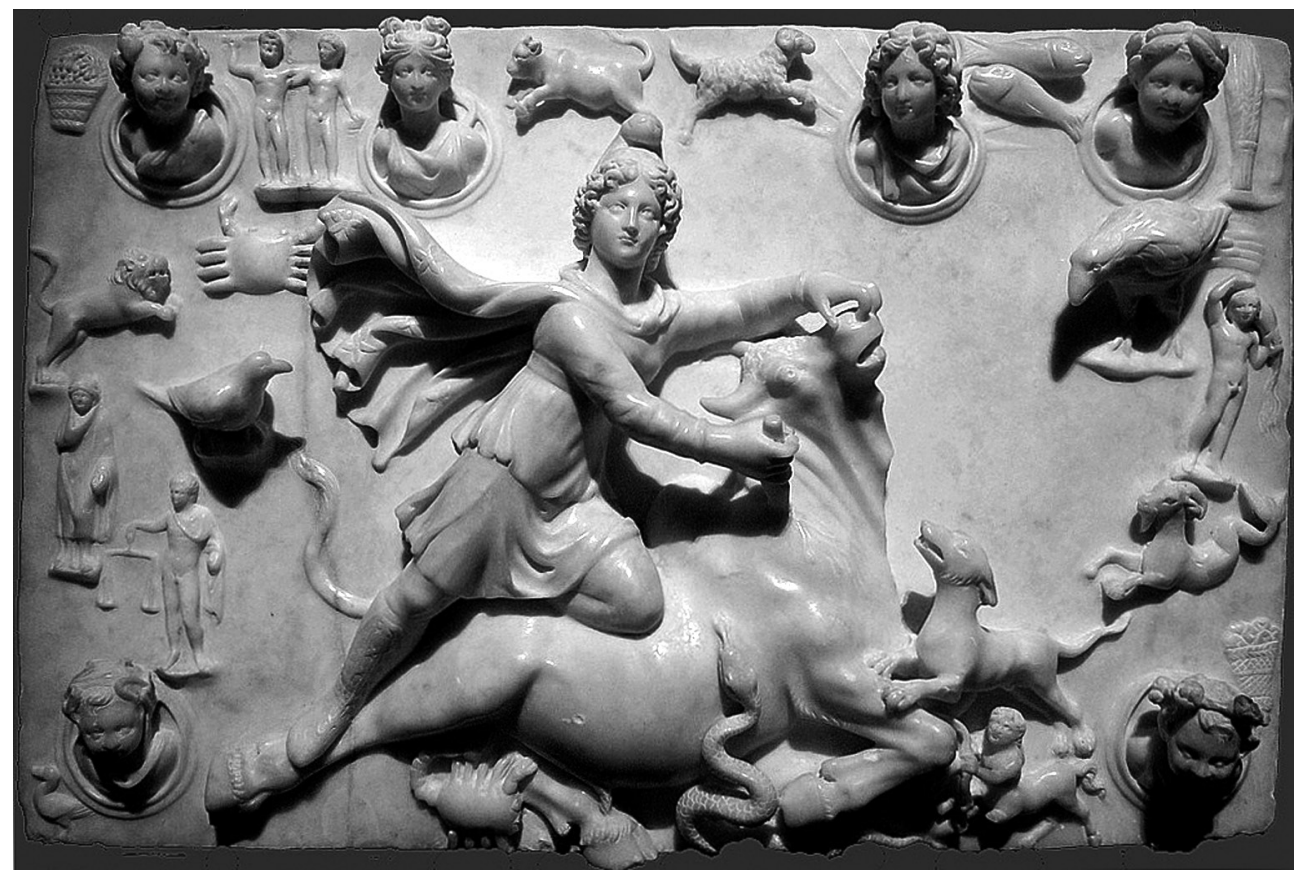

Fig. 1: Tauroctonia da Sidone. Parigi, Louvre (CIMRM 75).

Le intrusioni sono tuttavia molto più significative in altre due tauroctonie siriane. Nel rilievo recentemente donato da un ricco privato statunitense al Museo archeologico di Gerusalemme e pubblicato per la prima volta da Albert De Jong e quindi nuovamente commentato da Gordon, la sintassi compositiva è manifestamente diversa, direi unica per molti aspetti [fig. 2]. ${ }^{19}$ È come se l'ansia di includere scene secondarie del mito mitraico abbia prevalso sullo spazio a disposizione, sconfinando all'interno della grotta del sacrificio. Questo sconfinamento ha prodotto questa sorta di strani fumetti, a destra, ma soprattutto a sinistra della scena del sacrificio. Se tuttavia partiamo da ciò che circonda la grotta si vede una curiosissima inversione dei dadofori rispetto alle scene secondarie. Quando sono presenti entrambi - dadofori e scene laterali - normalmente i dadofori sono

19 De Jong 1997, 53-63; Gordon 2001b. 


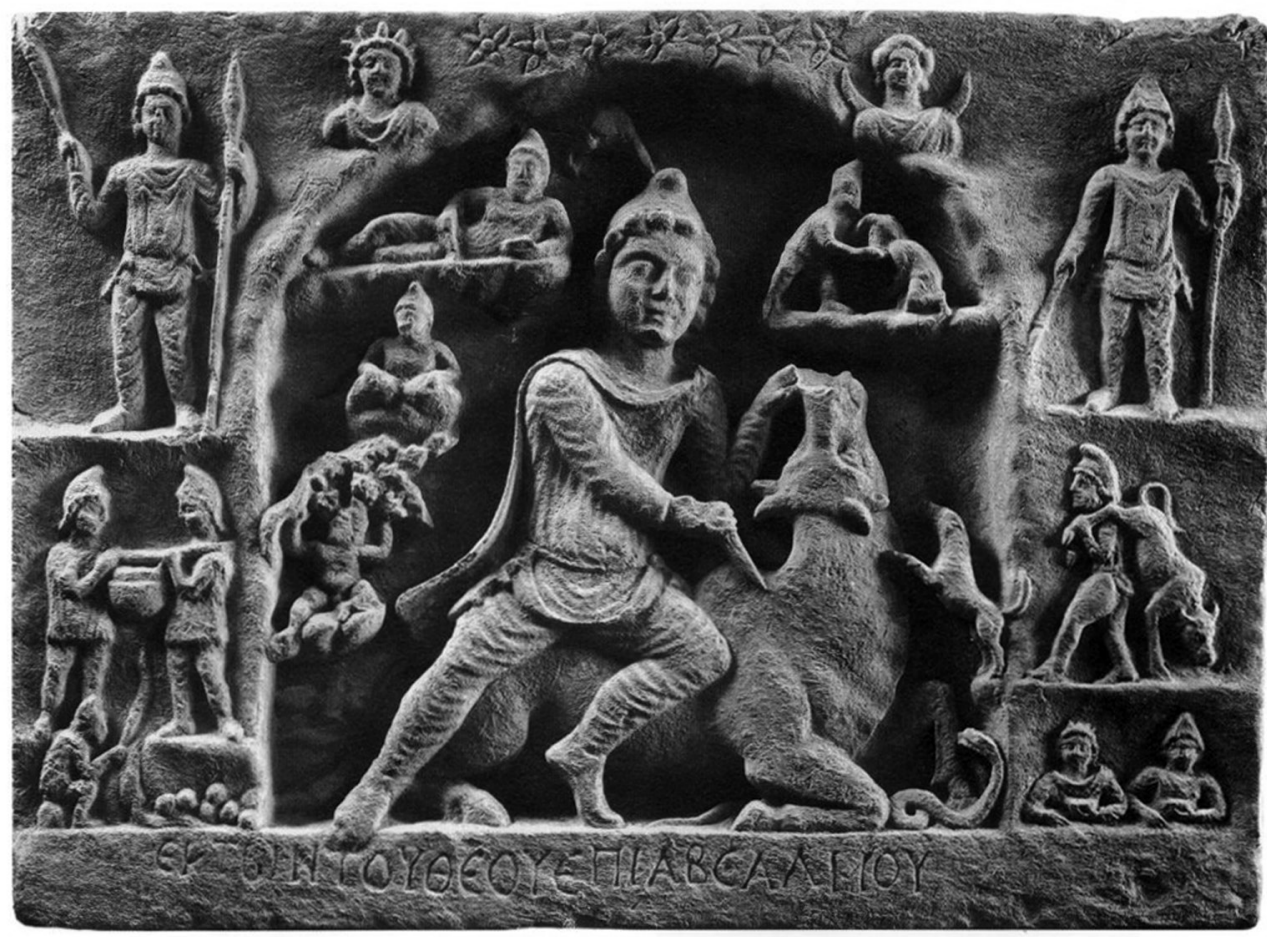

Fig. 1. Relief depicting the god Mitbras slaying a bull. Israel Museum Collection 97.95.19 Gift of Sam Merrin, New York, to American Friends of the Israel Museum

Fig. 2: Tauroctonia da Gerusalemme (da R.L. Gordon, A new Mithraic relief in the Israel Museum, Jerusalem, a summary of A. de Jong, A New Syrian Mithraic Tauroctony, Bulletin of the Asia Institute n. s. 11 (1997 [2000]), «Electronic Journal of Mithraic Studies» 2, 2001).

sempre o all'interno della grotta (sono infatti tra le figure normalmente ammesse), oppure se ne stanno ai lati, spesso fungendo nella sintassi compositiva da pilastri sui quali poggia la volta della grotta. Qui, al contrario, sono collocati in alto, Cautes a sinistra Cautopates a destra, simmetricamente affiancati da Sol e Luna, questi ultimi in posizione acroteriale rispetto al mistico spelaeum dove si svolge il dramma cosmico. I dadofori di questa complessa composizione hanno caratteristiche rispettivamente uniche o comunque molto rare: non hanno, come al solito, le gambe incrociate e sono dotati di lunghe lance, che ne fanno quasi degli 'dei armati', stando alla felice definizione di Henri Seyrig per caratterizzare una variegata categoria di divinità, spesso riscontrate nella Siria centro-settentrionale. ${ }^{20}$ In ambito mitraico l'unico confronto è, ancora una volta, con la rozza raffigurazione di Arsha-wa-Qibar. Nella posizione normalmente occupata dai dadofori, ai lati della grotta, si trovano quattro scene laterali, due per parte, più o meno simmetriche da un punto di vista compositivo, quelle mediane avendo molto più spazio in altezza rispetto a quelle inferiori. Due di queste scene sono prive di confronti, mentre un'altra, quella del tauroforo, presenta caratteristiche tali da differenziarla in modo notevole da tutti gli altri esempi noti. Tuttavia, parlando di sintassi compositiva, l'aspetto

${ }^{20}$ Seyrig 1970. 77-112; 1971, 67-70. 
più stupefacente è costituito dalla presenza di tre scene, ancora una volta tutte molto rare. Procedendo in senso orario e partendo da sinistra in basso, si vede un personaggio nudo e grassoccio, con il berretto frigio, con le braccia alzate verso una volta costituita da tralci e pampini d'uva. Si tratta senz'altro di una scena dell'infanzia di Mithra, che normalmente viene rappresentato uscente dalle rocce. ${ }^{21}$ Sopra si vede un personaggio forse ancora nudo e con berretto frigio, accovacciato su una roccia, le braccia appoggiate sulle cosce, immagine anch'essa unica nell'iconografia mitraica. Al di sopra lo stesso personaggio, caratterizzato dal berretto frigio, è rappresentato disteso su una kline in un atteggiamento mille volte riscontrato, ad esempio, su tanti sarcofagi sparsi nel mondo mediterraneo. Tiene qualche oggetto di difficilissima identificazione in entrambe le mani, e questa volta è certamente vestito alla persiana. Dall'altra parte, infine, Mithra, vestito alla persiana e con berretto frigio, sembra aiutare ad alzarsi un personaggio inginocchiato in segno di adorazione. In altre scene analoghe questo personaggio è Sol, qui si deve tuttavia notare come anche il personaggio inginocchiato sembrerebbe indossare un berretto frigio, anziché il più comune disco radiato.

Questa soluzione compositiva, d'inserire all'interno dello spelaeum scene che, quando s'incontrano, sono limitate al contorno della scena, è una soluzione rarissima. $\mathrm{Ne}$ conosco tre soli esempi, che non hanno nulla a che fare con la Siria.

Il primo proviene dalla Pannonia Inferiore, ed è una ben nota piccola lastrina di marmo, (appena 21 x 13-15 cm: CIMRM 1740), dove le dimensioni impediscono una sicura lettura delle scene, che appaiono appena abbozzate e prive di dettagli. Nonostante le dimensioni, sono presenti una scena nel registro superiore, e tre scene abbastanza ben riconoscibili nel registro inferiore. Ma quel che più c'interessa è quel che si vede sullo sfondo della tauroctonia: alle spalle di Cautopates un busto irriconoscibile, alle spalle di Cautes, in alto a destra due personaggi. È possibile forse individuare nei due busti i soliti Sol e Luna, mentre la posa del terzo, con le braccia alzate, sembrerebbe rinviare all'immagine della nascita di Mithra, come si riscontrava in basso a sinistra nella tauroctonia di Gerusalemme. Un altro rilievo, questa volta da Vienna e lì conservato (CIMRM 1650), lascia intravvedere, sullo sfondo della grotta, la scena del transitus, con Mithra che procede verso destra. ${ }^{22}$

La somiglianza con la soluzione compositiva nella tauroctonia di Gerusalemme è ancora maggiore nell'altro esempio a me noto. Si tratta di una celebre tauroctonia proveniente da Otricoli e oggi conservata ai Musei Vaticani (CIMRM 556, fig. 159). ${ }^{23}$ Qui, fatte le dovute differenze, la scena è invasa dai due dadofori, cosa non grave, uno dei quali, Cautopates, questa volta a sinistra, è rappresentato molto piccolo per fare spazio, alle sue spalle, alla scena di Mithra tauroforo (si noti in questa rappresentazione, rispetto a quella precedente, il senso da sinistra a destra anziché il contrario, inoltre Mithra è vestito anziché nudo). In alto a sinistra, però, è presente l'immagine di un giovane nudo che sembra uscire da una pietra. La figura così com'è è frutto di un pesante restauro, per cui non sono da prendere in considerazione l'aspetto della testa, la mancanza di berretto frigio né la posizione delle braccia.

${ }^{21}$ Neri 2000, 227-245; Heyner 2013, 219-229.

${ }^{22}$ Cfr. ora Gordon 2004, 259-283, in partic. 266, nota 48 (ad CIMRM 1740); Sicoe 2013, 285-302, in partic. 300 (ad CIMRM 1650).

${ }^{23}$ Ringrazio la collega Giovanna Bastianelli per avermi indicato la letteratura rilevante sulla provenienza di questo reperto: Pietrangeli 1942, 47-104, in partic. 52, 70, 89 e Pietrangeli 1978. 
Tuttavia l'intrusione più clamorosa all'interno del campo della grotta non è costituita dalle quattro scenette mitologiche presenti nello spelaeum raffigurato nella tauroctonia di Gerusalemme, bensì da quanto si può vedere all'interno della spelonca raffigurata nella tauroctonia maggiore di Dura Europos [fig. 3]. Qui, infatti, un buon terzo della scena risulta occupato da ben cinque personaggi che non hanno alcun riscontro in nessun altro monumento mitraico. Possiamo esser certi dell'identità di uno soltanto di questi.

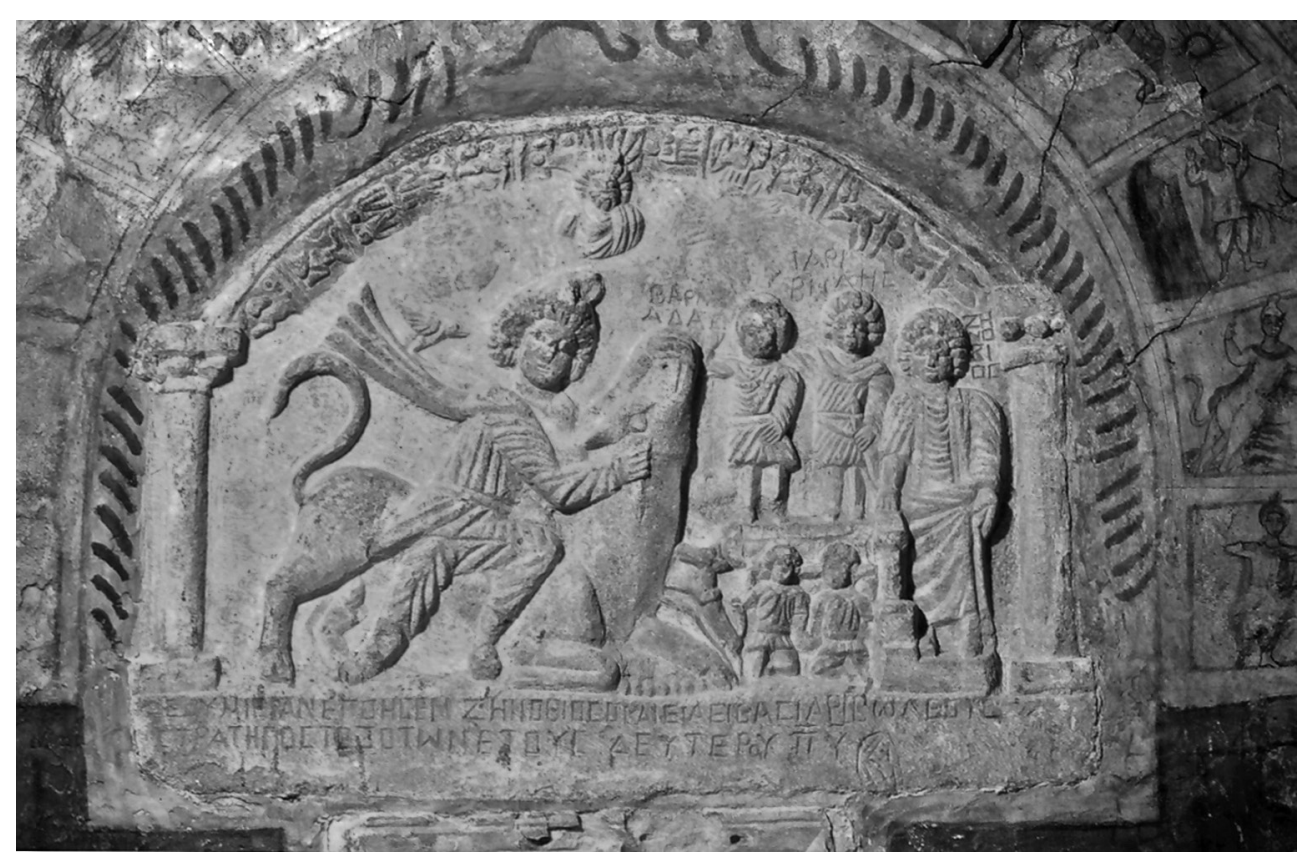

Fig. 3: Tauroctonia maggiore da Dura Europos (CIMRM 40) (Yale Art Gallery).

Infatti sulla base della scena vi è un'iscrizione greca su due righe che chiarisce data e dedicante: "Zenobios detto anche Eiaebas, figlio di Iaribol, strategos degli arcieri, ha fatto (questo rilievo) per il dio Mithra nell'anno 482 (dell'èra Seleucide = 170-1 d.C.)." La scena è inquadrata in un ambiente colonnato a volta. Lo spazio delimitato dall'ambiente colonnato è per circa un terzo occupato da questo pubblico del tutto straordinario in scene analoghe. Dagli angoli superiori, esterni alla volta, due personaggi, certamente Luna e Sol, assistono alla scena cruenta. La volta è decorata con i dodici segni dello zodiaco interrotti al centro dal busto di un personaggio barbato con kalathos. Mithra compie il gesto cruento nella consueta posizione, ma l'imperizia dello scultore fa sì che la gamba sinistra del dio, piegata nel gesto di schiacciare a terra l'animale morente, non sembri ottenere il suo scopo. Il braccio sinistro, che pugnala l'animale, è troppo lungo, ma, essendo piegato, giunge ugualmente sulla spalla del toro, il braccio destro, come al solito, solleva la tesa dell' animale, afferrandone saldamente le narici. Il toro è rappresentato con le zampe anteriori, l'una piegata col ginocchio a terra, l'altra tesa in avanti. Tra il ginocchio e lo zoccolo della zampa sinistra sono rappresentate sette piccole sfere allineate. Gli altri ovvi spettatori della tauroctonia, il corvo, il cane, il serpente, sono tutti rappresentati, ma in proporzioni veramente minuscole. 
È assente lo scorpione, qui come anche nella tauroctonia più piccola ritrovata accanto e, forse, in quella di Gerusalemme.

Per quanto riguarda i cinque personaggi che assistono alla scena, essi sono quattro disposti su due ordini, piccoli sotto, più grandi sopra, mentre un personaggio di dimensioni molto maggiori, togato e con lorica, è in piedi a sinistra. Delle didascalie in greco consentono d'identificare tre di questi personaggi. Quello più esterno, più grande e rappresentato mentre pone un'offerta su un braciere, è il committente del rilievo, cioè quel Zenobios strategos degli arcieri che si è già incontrato nell'iscrizione. Per quanto riguarda però la descrizione di questo pubblico di cinque persone preferisco citare la descrizione di Cumont, tradotta da Francis e ripresa verbalmente da Vermaseren (CIMRM 40):

Zenobius, the father, is pouring a libation on the altar. His two sons are standing on a ledge to his right and, according to the rule of isocephaly, they are represented as smaller than he. One of them, named Barnaadath, wears a simple tunic and raises his right hand with his palm turned forwards, his thumb extended, and his fingers doubled into his palm. The other seems to be making the same familiar gesture of adoration. He is dressed in a military costume and wears the paludamentum; his left hand rests on his sword, which hangs from a belt at his waist. Like his grandfather's, his name is Iariboles. Beneath Barnaadath and Iariboles we see two small kneeling figures. At first sight they might be taken to represent Atlantes supporting the ledge, but it is more likely that they are the grandchildren of Zenobius, placed below their parents. ${ }^{24}$

Si avrebbero dunque qui tre generazioni di mitraisti! Nonno, figli e nipoti sarebbero rappresentati mentre assistono a questa scena centrale del culto mitraico. Si deve tuttavia rilevare come i rapporti esistenti tra questi personaggi siano tutt'altro che sicuri e che sia arduo pensare a una raffigurazione familiare come questa in un contesto, quello militare, dove ancora non erano apertamente consentite relazioni familiari per i soldati in attività - anche se lo statuto giuridico dei militari inquadrati in unità ausiliarie appare meno definita di quella relativa ai legionari. Mi sembra pertanto intrinsecamente più plausibile l'ipotesi avanzata da Lucinda Dirven, che ipotizzava di vedere, nei tre registri del pubblico di questa tauroctonia, tre diversi gradi d'iniziati, ovvero un gruppo di commilitoni che condividevano la loro esperienza misterica all'interno della medesima conventicola ${ }^{25}$. Accanto al soggetto più importante, lo Zenobios strategos, e, ciò che più conta, committente della scultura, vi sarebbero quindi due personaggi - Iariboles e Barnaadath - che avranno avuto un ruolo di qualche rilievo nell'ambito di questa comunità religiosa e che non necessariamente dovevano essere legati a Zenobios da un legame familiare. Al di sotto di questi, vestiti con un abbigliamento apparentemente identico, vi sono due personaggi più piccoli, inginocchiati. Si tratterebbe, secondo questa lettura, di iniziati con un grado talmente basso da non meritare l'onore di avere una didascalia che li rendesse individualmente riconoscibili. Erano forse reclute che ancora dovevano sottoporsi a un qualche rituale d'iniziazione? Oppure erano iniziati del grado più infimo, dei

24 Cumont 1975, 167.

25 Dirven 1999, 271: "family ties were of little importance in Mithraic communities. Instead, worshippers of Mithras were a brotherhood of men who frequently shared the same profession. I therefore propose to identify the four figures as members of the Mithraic community." Per avvalorare ulteriormente questo rilievo basterà rilevare come in genere siano veramente esigue le attestazioni di legami familiari all'interno delle comunità mitraiche: cfr. Clauss 1992; Gordon 1994, 459-474. 
'corvi' nel gergo mitraico? È naturalmente possibile, e forse addirittura probabile. Fatto sta che Cumont li aveva paragonati a due Atlanti, che sorreggevano, con la loro presenza, il piano sul quale poggiavano i personaggi superiori.

Un elemento di difficoltà nella ricostruzione proposta da Dirven è rappresentato dall'onomastica dei due personaggi del registro superiore, Iariboles e Barnaadath. Le difficoltà non riguardano certo il primo dei due, che porta un nome teoforico molto bene attestato a Palmira. Un dio Yarhibôl era lì venerato con un tempio ed era associato alla sorgente Efqa, una vena di acqua sulfurea attorno alla quale si aggregò il nucleo originale della grande città carovaniera. Conseguentemente molti palmireni portavano quel nome; tuttavia il secondo nome, Barnaadath è, in questa forma, un hapax. ${ }^{26}$

Józef Milik spiegava il nome con una etimologia semitica: Bar-na-hadat "notre fils est neuf'. ${ }^{27}$ Più recentemente invece Dirven suggeriva un errore per Bar-hadad, 'dono di Hadad' ${ }^{28}$ Sono sempre poco incline a correggere i testi antichi, se non è proprio necessario, tuttavia si deve quanto meno tenere in considerazione una possibile etimologia iranica, non semitica, del nome: in questo caso il composto sarebbe da Farna-adath 'dono dello splendore/gloria' o qualcosa di simile. Il nostro Barnadat si andrebbe pertanto ad aggiungere ai tanti Farnace, Farnabazo etc. che ben conosciamo. In un ambiente fortemente semitico com'era quello di Dura Europos il suo nome si sarebbe pertanto 'semitizzato', per così dire, mutando l'aspirata iniziale in un modo perfettamente analogo a quanto è testimoniato da un passo delle Antichità giudaiche di Flavio Giuseppe $(11,207)$, molto opportunamente ricordato da Ferdinand Justi nel suo Iranisches

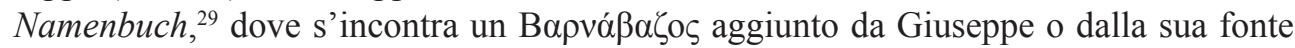
alla parafrasi di Esther II, $21 .{ }^{30}$ In entrambi i casi, in ambiente semitico, la radice iranica Farn- 'luce, splendore' sarebbe stata modificata in Bar-'figlio', suffisso comunissimo nell'onomastica semitica. I due personaggi anonimi della tauroctonia maggiore di Dura Europos portano quindi nomi di possibile derivazione uno semitica, l'altro iranica - ma del quale ultimo si era persa coscienza dell'iranicità, mutando appunto l'iranico farn- in un molto più semitico bar-. Ma c'è di più: scrivendo la sua sintesi - è vero ormai molto invecchiata - su La religion des Palmyreniens, James Février iniziava in modo sconsolato il paragrafo dedicato a Yarhibôl: "Le nom même de Yarhibôl sembre un défi à la science étymologique". ${ }^{31}$ Nonostante la prima parte del suo nome richiami infatti la luna per via di una radice YRH che rinvia sia alla Luna che al mese lunare, il dio palmireno è senz'altro una divinità solare, Jean Starcky concludeva quindi le sue considerazioni al riguardo cinquant'anni dopo in modo non meno desolato "On n'a pas trouvé de solution satisfaisant à cette énigme"! 32 Pur non potendo risolvere qui la questione, gioverà rilevare in conclusione come i due personaggi stanti, caratterizzati da un abbigliamento un

${ }^{26}$ Sull'onomastica palmirena è molto utile dal punto di vista metodologico Yon 2015, 11-18, che comunque, incentrato soprattutto su Zeugma, non tratta il nostro Barnaadath.

27 Milik 1972, 336-337.

${ }^{28}$ Dirven 1999, 266-267.

29 Justi 1895 , s.v.

${ }^{30}$ Sul passo cfr. Cassel 1888, 80, 303, 327-328; il problema del rapporto tra Giuseppe e le sue fonti, in particolare la $L X X$, è tema al quale non si può nemmeno accennare qui. Mi limito a segnalare Schürer 1973, in partic. vol. III.1, 506; vol. III.2, 718-722; Cohen 1979, in partic. 36.

${ }^{31}$ Février 1931, 81.

32 Starcky-Gawlikowski 1985, 95. 
po' diverso (Iaribol è armato, Barnaadath no), portano due nomi con radici che rinviano uno alla luna YRH l'altro alla luce FARN, e che nelle tauroctonie di Dura mancano i dadofori.

Il mitreo siriano scoperto più di recente, nel 1997, getta forse una nuova luce su tutta una serie di personaggi minori, difficilmente inquadrabili all'interno dell'immaginario mitraico così come ci si sforza di ricostruire, e che vengono spesso definiti, appunto, Atlanti, perché spesso rappresentati mentre sorreggono grandi sfere, oppure ripiani etc. Intendo lo straordinario mitreo rinvenuto sotto la grande chiesa di Hūarte, nel territorio di Apamea, nella Siria settentrionale, il luogo che ha rappresentato l'impegno di una vita dei coniugi Pierre e Maria Teresa Canivet. ${ }^{33} \mathrm{Mi}$ sia consentito su questo punto richiamare l'attenzione su un dato. Il sito di Hūarte era notissimo agli archeologi, ${ }^{34}$ ed era stato oggetto di una lunga serie di campagne di scavo durate quasi vent'anni, esattamente sull'edificio che obliterava il nostro mitreo. Se all'improvviso e inaspettatamente una volta non avesse ceduto sotto il peso di piogge straordinarie che si sono abbattute nell'area nell'inverno 1996-1997 il mitreo non sarebbe mai stato scoperto.

Sia come sia, la caratteristica straordinaria di questo mitreo risiede nelle meravigliose pitture che adornavano gran parte delle pareti del complesso ipogeo. ${ }^{35}$ Purtroppo la costruzione della soprastante chiesa cristiana ha comportato la distruzione completa di buona parte dell'alzato, e poche sono le immagini ancora leggibili nelle parti superiori, nessuna completa. Particolarmente lacunose sono le immagini che adornavano una parete dell'ingresso e i due pilastri della porta che immetteva all'interno della sala di culto. Nella prima era rappresentato un grande leone che si avventa su esserini neri che tentano invano di mettersi in salvo: uno di loro è rappresentato mentre viene dilaniato tra le fauci del leone. Sui pilastri invece sono rappresentate due figure stanti, che tengono per le briglie un grande cavallo bianco riccamente bardato, conformi all'iconografia dei Dioscuri, sporadicamente attestati in mitrei occidentali, come quello di Walbrook, a Londra. ${ }^{36}$ I protagonisti delle due scene sono rappresentati con un abito ricchissimo, chiaramente iranico. Uno di loro, quello meglio conservato e più leggibile, con la mano libera dalle briglie tiene alla catena un esserino nero. Si tratta di un mostriciattolo nudo, accoccolato, con una testa bifronte [fig. 4].

Qui, sulle 19 scene parzialmente ricostruibili che si trovano dipinte sugli strati d'intonaco più recenti del mitreo, ${ }^{37}$ strati che risalgono con precisione agli anni di Costanzo II e di Giuliano, sono raffigurati una quantità di omiciattoli neri, piccoli, mostruosi, accucciati negli angoli di sfavillanti scene di corte, oppure rappresentati mentre tentano invano di sfuggire dalle grinfie di enormi, terribili leoni. Ho descritto altrove questa scena detta 'dei Dioscuri' che si trova raffigurata, con un doppione simmetrico, sui pi-

33 Canivet - Canivet 1987.

34 Una recente mise-au-point sul sito: Gawlikowski 2013, 261-270.

35 La letteratura è data supra, nota 13 .

36 C. Martínez Maza (2013, 167-178) indaga le sculture e i reperti relativi ad altre divinità rinvenute nel mitreo londinese, in un deposito interrato verosimilmente nei primi decenni del quarto secolo, e sparsi nella struttura. Si tratta di un Serapide, di una Minerva e di un Saturno, oltre a un gruppo bacchico. Non viene preso in considerazione il Dioscuro, che venne ritrovato all'esterno della struttura, a circa due metri di distanza e che è oggi conservato e visibile al Museum of London.

37 Gawlikowski 2007, 352. 
lastri che immettevano nello spelaeum. ${ }^{38} \mathrm{Se}$, come sono convinto, questi esserini neri devono intendersi come una rappresentazione visiva delle passioni e dei vizi dell'uomo, quello che il mitreo di Hūarte ci fa conoscere è molto di più di una nuova scena finora non testimoniata del mito del dio tauroctono. Per la prima volta appare con tutta evidenza la dimensione etica del mitraismo. ${ }^{39}$

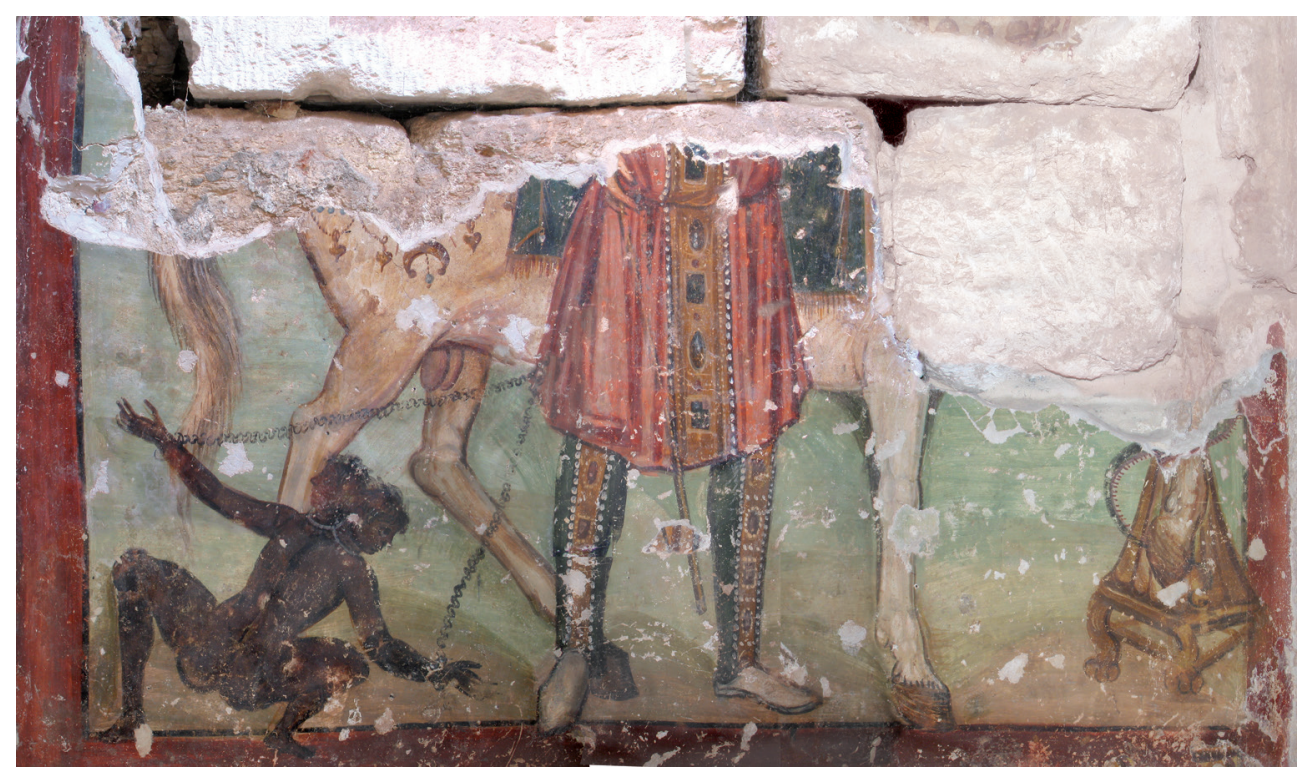

Fig. 4: Mitreo di Ḥūarte, scena 'del Dioscuro' (foto M. Gawlikowski).

La stessa dimensione etica è riscontrabile in un'altra scena, dipinta subito a sinistra della nicchia della cella, in un luogo, cioè, della massima importanza del nostro mitreo. All'interno di una riquadratura di color rosso mattone sono rappresentate le grandi mura difensive di una città, un monotono reticolo grigio interrotto solamente da una grande porta nera [fig. 5]. Al di sopra delle mura si vede una fila di teste molto grandi, mostruose, irsute, digrignanti i denti. Una di queste teste è rotolata sotto le mura, nell'angolo in basso a destra. Ogni testa, compresa quella caduta, è raggiunta da una linea gialla che la colpisce più o meno esattamente sulla fronte, partendo dall'alto. Nonostante il fatto che gli occhi delle teste siano stati tutti volontariamente deturpati durante la distruzione del mitreo da parte dei cristiani che costruirono sopra la chiesa, è facile immaginare che gli sguardi fossero rivolti nella direzione da cui provengono i raggi gialli.

Trovo veramente sorprendente la spiegazione di questa scena fornita da Gordon: secondo questo studioso dal momento che "de chaque crâne sort une lance ou une flèche. Il s'agit donc de soldats ou de prisonniers morts". ${ }^{40}$ Quindi, dopo aver ricordato quanto fosse comune la pratica della decapitazione, conclude affermando che, a suo parere, in questa scena vi sarebbe il concorso di due stereotipi, comuni nelle arti minori o nelle bot-

\footnotetext{
38 Gnoli 2009, 215-234.

39 Gnoli, in corso di stampa.

40 Gordon 2001a, 106-107.
} 


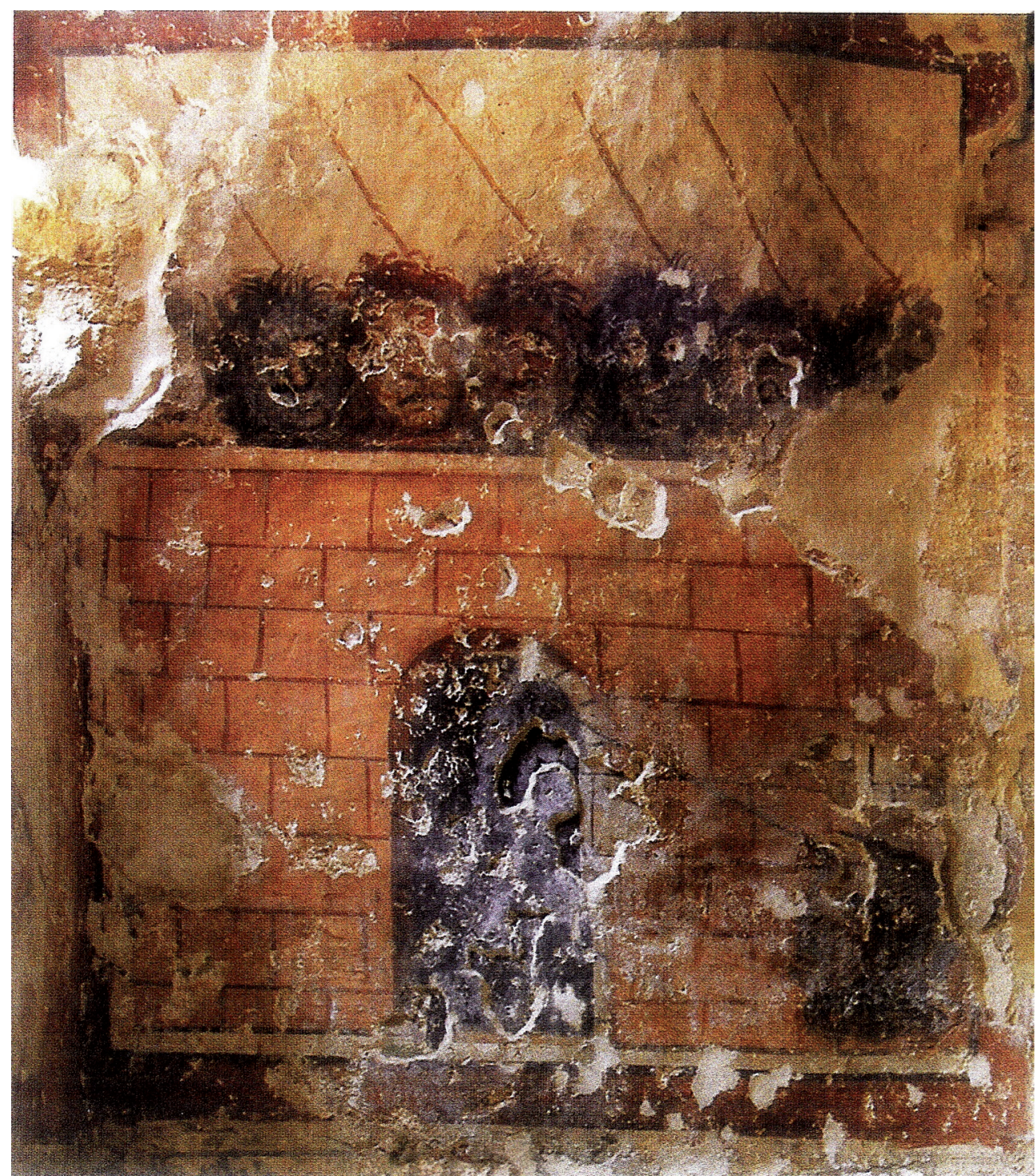

Fig. 5: Mitreo di Ḥūarte, 'la città delle tenebre' (foto M. Gawlikowski).

teghe artigiane della Siria tardoantica: 1. La rappresentazione di barbari sulle mura, della quale non può citare che un solo esempio, del tutto diverso dal nostro; 2 . Le venationes nell'anfiteatro, dove varie fiere sono rappresentate trafitte da una quantità di dardi.

Mi sembra sia inutile contestare questa lettura così forzata, tanto più strana in quanto formulata da uno dei maggiori esegeti riconosciuti del mitraismo romano. Altrettanto curiosa mi sembra l'idea avanzata da $\operatorname{Dirven}^{41} \mathrm{che}$, partendo dall'idea che la luce che

${ }^{41}$ Dirven, in corso di stampa. Ringrazio la collega per avermi permesso di leggere questo lavoro non ancora terminato. 
colpisce le teste potrebbe in realtà essere emessa da esse, propone di identificare le teste di Hūarte con gli arconti manichei. Nulla sostiene questa ipotesi, non fosse altro che per il motivo che, secondo la mitologia manichea, gli arconti emetterebbero la luce come liquido seminale!

Ho già manifestato altrove come io mi trovi in pieno accordo con Gawlikowski quando afferma che "the scene is strikingly Zoroastrian in spirit". ${ }^{42}$ In effetti le teste, come presentate nell'affresco, non presuppongono l'esistenza di un corpo, ma sembrano essere entità autonome e complete, cosa perfettamente corrispondente allo stato totalmente spirituale dei demoni zoroastriani, dove corporeità e materialità, gêtīg, sono esclusivo appannaggio delle forze del bene. Sempre nella tradizione zoroastriana, già nei testi avestici si trova la distinzione tra 'teste buone' e 'teste cattive', quelle dei demoni delle persone malvagie. Lì il termine avestico per 'testaccia' kamərəda- è associato al nome del demone Arəzūra- per indicare un toponimo specifico, cioè il luogo dove i demoni si danno convegno e che viene ritenuto la porta dell'inferno. ${ }^{43} \mathrm{La}$ rappresentazione del mitreo di Ḥuarte soddisfa queste caratteristiche esclusive della tradizione zoroastriana: 'testacce' di demoni raggruppate sulla sommità di un edificio che presenta una porta nera che conduce all'inferno. Solo la luce di Ohrmazd, rappresentata dalle linee gialle che colpiscono ciascuna delle testacce, può sconfiggere questi orrendi demoni arimanici. La spiegazione di Gordon può spiegarsi solo con il tentativo disperato di far rientrare questa scena all'interno del nuovo paradigma ermeneutico.

Il rinvenimento del mitreo di Ḥuarte, studiato senza preconcetti e senza tesi da difendere, costringe a rielaborare molte cose che, secondo il nuovo paradigma, sembravano ormai acquisite.

\section{Una cosmogonia mitraica?}

Tornando ora a occuparci delle due più rilevanti tauroctonie siriane, quella di Gerusalemme e quella maggiore di Dura Europos, è stato giustamente notato che i due monumenti offrono importanti punti di contatto. Quello più significativo è a mio parere una esplicitazione del contenuto cosmogonico del sacrificio mitraico. Benché si sia recentemente tentato di negare qualsiasi correlazione tra il sacrificio del toro e l'ordine cosmico, mi sembra difficile spiegare altrimenti la presenza di sette elementi globulari che compaiono sia nella tauroctonia di Gerusalemme, sia su quella di Dura Europos. ${ }^{44}$ La prima delle scene che, al di fuori della grotta s'incontra in basso a sinistra nella tauroctonia di Gerusalemme mostra un personaggio accucciato, Mithra a giudicare dal consueto abbigliamento, seduto su una roccia a sua volta globulare, il busto leggermente proteso in avanti, mentre tiene con la sinistra un oggetto allungato difficilmente individuabile. ${ }^{45}$

42 Gawlikowski 2007, 355; cfr. Gnoli 2009, 229-232; e Gnoli, in corso di stampa.

43 Bartholomae 1904, 202; Asmussen 1987, 691-692.

44 Cfr, anche quanto scritto supra, p. 7.

45 De Jong $(1997,56)$, pensava piuttosto a Saturno, sulla base però di altre raffigurazioni un po' diverse da quella commentata, e avanzava fra molti dubbi l'ipotesi che l'oggetto tenuto con la destra fosse una '(clumsy)' harpè. Mi sembra migliore l'interpretazione di Gordon (2001b), che individua nella scena la presenza di una sorgente che sgorga dalla roccia sulla quale si appoggia la scena superiore (l'acqua sarebbe 
Gordon è giustamente perplesso nell'interpretazione di questa scena, posta con ogni evidenza all'inizio di un qualche racconto che procede fino all'angolo in basso a destra del rilievo, dove si trovano raffigurati Mithra e Sol mentre libano: come spiegare le sette sfere ammassate alla rinfusa nella scena, quasi fossero delle grandi biglie con le quali si trastulla Mithra? A mio parere ha certamente ragione De Jong quando, su questo particolare, richiama un altro gruppo di altri piccoli sette globuli, che questa volta compaiono perfettamente allineati tra le zampe del toro morente nella tauroctonia maggiore di Dura Europos. La presenza di sette elementi uguali e allineati a margine delle tauroctonie è tutt'altro che rara. Si ritrovano con una certa frequenza sette altari allineati al di fuori della grotta, spesso al di sopra; oppure possono trovarsi sette astri allineati nella stessa posizione. Quello che differenzia le due tauroctonie siriane qui esaminate (e in particolare quella di Gerusalemme) è l'inserimento delle sette sfere nella vicenda della tauroctonia, dentro, cioè, lo spazio mistico dello spelaeum, che, come si è visto, nelle raffigurazioni siriane si presta ad 'accettare' di buon grado elementi che altrove sono rarissimi.

\section{Accenni di una economia del mitreo}

C'è tuttavia un aspetto che differenzia la tauroctonia di Gerusalemme in un modo tale da renderla del tutto unica. Si tratta della semplicissima iscrizione che accompagna il

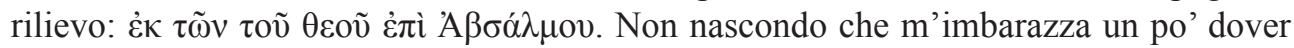
dire che questo testo significa che il rilievo è stato fatto da Absalmos con i fondi del dio, eppure è necessario. L'editore De Jong ha dovuto faticare non poco per giungere alla lambiccata traduzione: "Because of the things [received] from the god; in the time of Absalmos". De Jong era naturalmente ben consapevole dell'ovvio significato della frase, eppure:

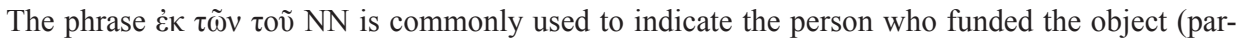

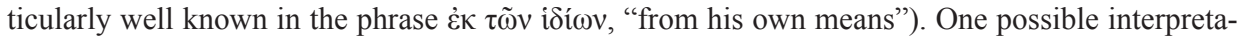
tion would therefore be "funded by the god," but this does not make much sense and is unparalleled in Mithraic works of art. ${ }^{46}$

Ancora una volta, dunque, ci troviamo di fronte all'esigenza primaria di 'normalizzare' un testo eterodosso, che mal s'integra nel nuovo paradigma ermeneutico. Secondo De Jong l'unica ragione per cui questo testo, a differenza di dozzine di altri testi analoghi provenienti da tutto il Vicino Oriente, non potrebbe significare che il rilievo è stato fatto "a spese del patrimonio del dio" è che non vi sarebbero altre attestazioni analoghe in testi mitraici. Invece d'intedere il rilievo di Gerusalemme come la prima attestazione di un patrimonio connesso a un tempio mitraico si preferisce una traduzione molto più complicata, basata su esempi incerti e lontanissimi nel tempo e nello spazio. Ma c'è di più. De Jong interpreta غ̇สì A $\beta \sigma \alpha ́ \lambda \mu o v$ come una formula di datazione. Si tratterebbe di un'ipotesi a mio parere molto attraente, se fosse probabile, perché consentirebbe di progredire sulla

l'oggetto allungato di difficile lettura). Molto utile è la notizia che egli riporta in sede di commento, cioè il fatto che, a detta della compianta Ingeborg Huld-Zetsche, si sarebbero ritrovate nel mitreo di Dormagen ben dodici pietre sferiche di dimensioni decrescenti che non sono state menzionate in CIMRM.

${ }^{46}$ De Jong 1997, 58. 
vexata quaestio della possibile esistenza di perduti testi mitraici. ${ }^{47}$ Temo tuttavia che anche la seconda parte di questa brevissima iscrizione sia molto meno rivelatrice di quanto ipotizzato da De Jong. In un articolo quasi dimenticato pubblicato nel 1912 sui Transactions and Proceedings of the American Philological Association uno dei maggiori esperti di epigrafia greca e latina di Siria, William Kelly Prentice, indagò a fondo l'uso delle preposizioni impiegate sulle iscrizioni greche per indicare il completamento di edifici, restauri etc. da parte di quegli strani personaggi, tipicamente siriani, noti col nome di 'magistrati di villaggio'. ${ }^{48}$ Per quanto riguarda l'uso di غ̇ंì + genitivo Prentice notava che nelle iscrizioni siriane questa frase designava sempre l'ufficiale di rango più

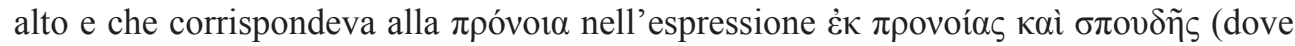
la $\sigma \pi 0 v \delta \eta ́$ veniva invece resa tramite l'impiego di $\delta$ ió più genitivo). Ripeto ancora una volta: questa iscrizione non può significare altro che il rilievo era stato fatto da Absalmos con i fondi del dio.

Anziché tentare di 'normalizzare' questo testo e di ricondurlo su un sentiero più comodo e meglio battuto è a mio parere il caso di valorizzarlo e di connetterlo, se possibile, con qualcos'altro nel campo dell'organizzazione del culto di Mithra, quantomeno in Siria. La ricerca non è difficile. Esiste un altro mitreo siriano che ha caratteristiche uniche: si tratta del santuario di Ša āra un sito nell'Hawrān, località agricola posta non lontano dalla grande arteria militare della Strata Diocletiana a sud di Damasco studiato negli anni 1990 da Mikaël Kalos. ${ }^{49}$ Del mitreo non rimane altro oggi che l'arco che circondava la cripta nella cella del tempio, sufficiente comunque, con i scarsi resti della tauroctonia, ad identificare la funzione del tempio in maniera inequivoca. Quel che più conta però è che il piccolo complesso cultuale era direttamente connesso con un altro edificio per il quale gli archeologi hanno potuto provare una funzione economica. Questa associazione tra tempio mitraico ed edificio produttivo, unica finora a quanto è dato conoscere per quanto riguarda i mitrei, contribuisce a chiarire meglio il senso dell'iscrizione posta sotto la tauroctonia di Gerusalemme.

\section{Conclusioni}

Mi sembra che tutto quanto scritto finora, la cronologia estremamente antica dei monumenti mitraici di Siria, le particolarità compositive e tematiche presenti nei pochi monumenti rimasti, il ruolo esplicitamente ricoperto da peculiari dottrine mazdaiche negli affreschi di Ḥuarte, la nuova attestazione di una qualche forma di economia connessa ai luoghi di culto di Mithra, debba indurre a una maggiore prudenza negli studiosi, e che si debba prendere in considerazione, almeno come ipotesi di lavoro, una facies orientale del mitraismo romano, lasciando per ora impregiudicato il problema dei rapporti tra quest'ultima e la meglio nota facies occidentale.

${ }^{47}$ Si tratta di un tema molto delicato per tutta l'interpretazione del culto misterico di Mithra. Cfr. da ultimo, su questo, le utili considerazioni di J. Alvar (2008, 75-76). Per una visione estrema, tendente a negare spessore a qualsiasi 'dottrina' mitraica, cfr. le opere citate supra, n. 17.

${ }_{48}$ Prentice 1912, 113-123.

${ }^{49}$ Kalos 2001, 229-277. 
BIBLIOGRAFIA

Alvar, J. (2008), Romanising Oriental Gods: Myth, Salvation and Ethics in the Cults of Cybele, Isis and Mithras, Leiden-Boston.

Asmussen, J.P. (1987), Arzūr s.v., Encyclopedia Iranica II: 691-692.

Bartholomae, C. (1904), Altiranisches Wörterbuch, Strassburg.

Beck, R. (1984), Mithraism since Franz Cumont, $A N R W$ II,17.4: 2102-2115.

Beck, R. (1998), The Mysteries of Mithras: A New Account of their Genesis, JRS 88: 115-128.

Beck, R. (2000), Ritual, Myth, Doctrine, and Initiation in the Mysteries of Mithras: New Evidence from a Cult Vessel, JRS 90: 145-180.

Beck, R. (2004a), Beck on Mithraism: Collected Works with New Essays, Aldershot-Burlington.

Beck, R. (2004b), Whose Astrology? The Imprint of Ti. Claudius Balbillus on the Mithraic Mysteries, in: R. Beck, Beck on Mithraism: Collected Works with New Essays, Aldershot-Burlington: $323-329$.

Beck, R. (2006), The Religion of the Mithras Cult in the Roman Empire: Mysteries of the Unconquered Sun, Oxford.

Bianchi, U. (ed.) (1978), Mysteria Mithrae: Atti del Seminario internazionale su la specificità storicoreligiosa dei misteri di Mithra, con particolare riferimento alle fonti documentarie di Roma e Ostia, Roma e Ostia 28-31 marzo 1978, Leiden.

Blakely, J.A. (1987), The Joint Expedition to Caesarea Maritima, Excavation Reports: The Pottery and Dating of Vault, 1: Horreum, Mithraeum and Later Uses, Lewiston, NY.

Bongard-Levin, G.M. et al. (2007), Mongolus Syro salutem optimam dat: la corréspondence entre Mikhaïl Rostovtzeff et Franz Cumont, Paris.

Bull, R.J. (1978), The Mithraeum at Caesarea Maritima, in: J. Duchesne-Guillemin, Études mithriaques: Actes du $2^{\mathrm{e}}$ Congrès international, Téhéran, du $1^{\mathrm{er}}$ au $8^{\mathrm{e}}$ septembre 1975 (Acta Iranica 17. Première série: Actes de congrès 4), Leiden 1978: 75-89.

Campbell, L.A. (1968), Mithraic Iconography and Ideology, Leiden.

Canivet, P., Canivet, M.T. (1987), Hūarte, sanctuaire chrétien d'Apamène, IV-VI s., Paris.

Cassel, P. (1888), An Explanatory Commentary on Esther (Engl. transl. by A. Bernstein), Edinburgh.

Chabiera, A., Parandowska, E., Troclimowicz, A. (2004), Paintings from the Hawarte Mithraeum in Syria, in: L. Borhy, S. Palágyi, M. Magyar (éd.), Plafonds et voûtes à l'époque antique: actes du VIII ${ }^{\mathrm{e}}$ Colloque Internationale de l'Association Internationale pour la Peinture Murale Antique (AIPMA) 15-19 mai 2001, Budapest-Veszprém, Budapest: 321-326.

Clauss, M. (1992), Cultores Mithrae: die Anhangerschaft des Mithras-Kultes, Stuttgart.

Cohen, S.J.D. (1979), Josephus in Galilee and Rome: His Vita and Development as a Historian, Leiden. Cumont, F. (1896-1899), Textes et monuments figurés relatifs aux mystères de Mithra, t. I-II, Paris.

Cumont, F. (1902), Les mystères de Mithra, Paris.

Cumont, F. (1906), Les religions orientales dans le paganisme romain, Paris.

Cumont, F. (1933), Deux monuments des cultes solaires, Syria 14: 381-395.

Cumont, F. (1975), The Dura Mithraeum, in: J.R. Hinnels, Mithraic Studies: Proceedings of the First International Congress of Mithraic Studies, vol. I, Manchester 1975: 151-214.

De Jong, A. (1997), A New Syrian Mithraic Tauroctony, Bulletin of the Asia Institute, n. s., 11: 53-63.

Dirven, L. (1999), The Palmyrenes of Dura-Europos: A Study of Religious Interaction in Roman Syria, Leiden.

Dirven, L. La lotta tra la Luce ele Tenebre nel Mitreo di Huarte. Testimonianze affrescate sull 'interazione mitraico-manichea nella Siria tardo antica (in corso di stampa).

Duchesne-Guillemin, J. (éd.) (1978), Études mithriaques: Actes du $2^{\mathrm{e}}$ Congrès international, Téhéran, $d u 1^{\text {er }}$ au $8^{\mathrm{e}}$ septembre 1975 (Acta Iranica 17. Première série: Actes de congrès 4), Leiden.

Février, J.G. (1931), La religion des palmyréniens, Paris. 
Gagé, J. (1968), Basileia: les Cesars, les rois d'Orient et les mages, Paris.

Gatier, P.-L. (2001), Le site de 'Arsha wa-Qibar, Topoi 11: 175-182.

Gawlikowski, M. (2000), Un nouveau mithraeum récemment découvert à Huarté près d'Apamée, CRAI: 161-171.

Gawlikowski, M. (2007), The Mithraeum at Hawarte and its Paintings, JRA 20: 337-361.

Gawlikowski, M. (2013), Haouarté, un village d'Apamène, in: Villes et campagnes aux rives de la Méditerranée. Hommages à Georges Tate, Paris: 261-270.

Gawlikowski, M. (2014), Zoroastrian Echoes in the Mithraeum at Hawarte, Syria, ARAM 26: 109-117.

Gnoli, T. (2009), Giuliano e Mitra, AnTard 17: 215-234.

Gnoli, T. (2010), Mithras and the Stars: A Note, in: M. Macuch, D. Weber, D. Durkin-Meisterernst (eds.), Ancient and Middle Iranian Studies: Proceedings of the $6^{\text {th }}$ European Conference of Iranian Studies, held in Vienna, 18-22 September 2007, Wien: 77-86.

Gnoli, T. (2016), The Mithraeum of Dura-Europos: New Perspectives, in: T. Kaizer (ed.), Religion, Society and Culture at Dura-Europos, YClS 38: 146-164.

Gnoli, T. Ethics in Mithraism, in: P. Johnston, A. Mastrocinque (a cura di), Acta del Symposium Peregrinum 2016, Tarquinia (in corso di stampa).

Gordon, R.L. (1975), Franz Cumont and the doctrines of Mithraism, in: J.R. Hinnels, Mithraic Studies: Proceedings of the First International Congress of Mithraic Studies, vol. I, Manchester: 215-248.

Gordon, R.L. (1994), Who Worshipped Mithras?, JRA 7: 459-474.

Gordon, R.L. (2001a), Trajets de Mithra en Syrie romaine, Topoi 11: 77-136.

Gordon, R.L. (2001b): A New Mithraic Relief in the Israel Museum, Jerusalem, Electronic Journal of Mithraic Studies 2.

Gordon, R.L. (2004), Small and Miniature Reproductions of the Mithraic Icon: Reliefs, Pottery, Ornaments and Gems, in: M. Martens, G. De Boe (eds.), Roman Mithraism: The Evidence of the Small Finds: Papers of the International Conference, Tienen 7-8 November 2001, Bruxelles: 259-283.

Gordon, R.L. (2007), Mithras in Doliche: Issues of Date and Origin, JRA 20: 602-610.

Griffith, A.B. (2014), Dead Religion, Live Minds: Memory and Recall of the Mithraic Bull-Slaying Scene, Journal of Cognitive Historiography 1: 72-89.

Hinnels, J.R. (ed.) (1975), Mithraic Studies: Proceedings of the First International Congress of Mithraic Studies, vol. I, Manchester: 151-214.

Heyner, R. (2013), ‘Aus dem Felsen geboren ...’ Die Ikonographie des Mithras-Kultes, in: Imperium der Götter: Isis, Mithras, Christus. Kulte und Religionen im Römischen Reich, Exhibition at Karlsruhe, Karlsruhe: 219-229.

Hopfe, L.M. (1990), Mithraism in Syria, ANRW II,18.4: 2216-2234.

Justi, F. (1895), Iranisches Namenbuch, Marburg.

Kalos, M. (2001), Un sanctuaire de Mithra inédit en Syrie du Sud, Topoi 11: 229-277.

Majcherek, G. (2003), Hawarte: Excavation and Restoration Work in 2003, Polish Archaeology in the Mediterranean 15: 325-334.

Martin, L.H. (2005), Performativity, Discourse and Cognition: 'Demythologizing' the Roman Cult of Mithras, in: W. Braun (ed.), Rhetoric and Reality in Early Christianities, Waterloo: 187-217.

Martínez Maza, C. (2013), Los dioses sunnaoi del mitreo di Walbrook, Mythos 7: 167-178.

Mastrocinque, A., (2017), The Mysteries of Mithras. A Different Account, Tübingen.

Merkelbach, R. (1984), Mithras, Königstein/Ts.

Milik, J.T. (1972), Dédicaces faites par des dieux (Palmyre, Hatra, Tyr) et des thiases sémitiques à l'époque romaine, Paris.

Neri, I. (2000), Mithra petrogenito: origine iconografica e aspetti cultuali della nascita dalla pietra, Ostraka 9: 227-245.

Nilsson, M.P. (1950), Geschichte der griechischen Religion, II: Die hellenistische und römische Zeit, München.

Parandowska, E. (2002), Hawarte: Conservation of a Mural, Polish Archaeology in the Mediterranean 14: 295-299. 
Pietrangeli, C. (1942), Lo scavo pontificio di Otricoli, Rendiconti della Pontificia Accademia di Archeologia 19: 47-104.

Pietrangeli, C. (1978), Otricoli: un lembo dell'Umbria alle porte di Roma, [Narni].

Prentice, W.K. (1912), Officials Charged with the Conduct of Public Works in Roman and Byzantine Syria, TAPA 43: 113-123.

Schürer, E. (1973), The History of the Jewish People in the Age of Jesus Christ (175 B.C.-A.D. 135), eds. G. Vermes, F. Millar, Edinburgh.

Schütte-Maischatz, A., Winter, E. (2001), Die Mithräen von Doliche: Überlegungen zu der ersten Kultstätten des Mithras-Mysterien in der Kommagene, Topoi 11: 149-173.

Schütte-Maischatz, A., Winter, E. (2004), Doliche - eine kommagenische Stadt und ihre Götter: Mithras und Iupiter Dolichenus, Bonn.

Seyrig, H. (1970), Les dieux armés et les Arabes en Syrie (Antiquités syriennes 89), Syria 47: 77-112. Seyrig, H. (1971), Les dieux syriens en habit militaire, AAAS 21: 67-70.

Sicoe, G.D. (2013), Lokalproduktion und Importe. Der Fall der mithraischen Reliefs aus Dakien, in: Imperium der Götter: Isis, Mithras, Christus. Kulte und Religionen im Römischen Reich, Exhibition at Karlsruhe, Karlsruhe: 285-302.

Starcky, J., Gawlikowski, M. (1985), Palmyre, Paris.

Wendt, H. (2016), At the Temple Gates: The Religion of Freelance Experts in the Early Roman Empire, New York (non vidi).

Vermaseren, M.J. (1956), Corpus Inscriptionum et monumentorum religionis Mithriacae, Nijhoff.

Vermaseren, M.J. (1963), Mithras, the Secret God, London.

Vermaseren, M.J. (1971), Mithriaca I: The Mithraeum at S. Maria Capua Vetere, Leiden.

Vermaseren, M.J. (1974), Mithriaca II: The Mithraeum at Ponza, Leiden.

Vermaseren, M.J.(1982), Mithriaca III: The Mithraeum at Marino, Leiden.

Yon, J.-B. (2015), L'onomastique en Syrie orientale et en Mésopotamie à l'époque romaine, in: C. Abadie-Reynal, J.-B. Yon (éd.), Zeugma VI. La Syrie romaine. Permanences et transferts culturels, Lyon: 11-18. 